\title{
Possible Health Hazards Associated with the Use of Toxic Metals in Semiconductor Industries
}

\author{
Swaran J.S. FlorA \\ Division of Pharmacology and Toxicology, Defence Research and Development Establishment, Gwalior, India
}

\begin{abstract}
Possible Health Hazards Associated with the Use of Toxic Metals in Semiconductor Industries: Swaran J.S. FLoRA. Division of Pharmacology and Toxicology, Defence Research and Development Establishment, Gwalior, IndiaGallium Arsenide (GaAs), Indium Arsenide (InAs) and Indium Phosphide ( $\ln P$ ) are the intermetallic compounds that are recognised as a potential health risk to workers occupationally exposed to their dust. Exposure to these semiconductor compounds in the microelectronic industry can occur during the preparation of material, cleaning and maintenance operations for quartz glassware and during cleaning of the reactor. The toxic effect of the intermetallic semiconductors appears to occur due to inhalation or oral exposure and may result in poisoning. Assessment of risk to workers engaged in $\mathrm{GaAs} / \operatorname{In} \mathrm{As}$ production is difficult due to the lack of data on the toxicity of these compounds. Their toxicity is mainly estimated on the basis of inorganic arsenic because it is now well known that GaAs and InAs dissociate into their constitute moieties and exert adverse effects on the haematopoietic and immune systems. As their toxicity is still not very well understood the treatment also remains to be elucidated.
\end{abstract}

(J Occup Health 2000; 42: 105-110)

Key words: Gallium arsenide, Indium arsenide, Indium phosphide, Metal distribution, Biochemical changes, Experimental evidence, Chelation treatment

In the defence microelectronic industry, silicon was previously the substrate predominantly used, but in recent times a number of other semiconductor materials (particularly III-V intermetallic semiconductors) have been introduced which have an increasing number of applications. Gallium arsenide (GaAs) is one of the substrates which have superior properties for making high

Received Aug 17, 1999; Accepted Jan 8, 20010

Correspondence to: S.J.S. Flora, Division of Pharmacology and Toxicology: Defence Research and Development Establishment. Jhansi Road, Gwalior-474002, India frequency devices, and photon emitters followed by gallium phosphide ( $\mathrm{GaP}$ ), indium phosphide ( $\mathrm{InP}$ ) and indium arsenide (InAs). These III-V intermetallic scmiconductors are crystalline and intermetallic compounds. They are prepared by condensing vapours of the elemental forms of the metalloids. They have found distinct and continually expanding application in the semiconductor industry. Such a demand can be expected to result in an increase in the production and processing of ingots and wafers, which has the potential to expose much of the semiconductors industry to these toxic metals. The chemical from of the intermetallic does not appear to be as important for toxicology as the chemical forms of its dissolution products. In the manufacture of these semiconductors there are four major steps:crystal growth, wafer processing, epitaxy production and device fabrication. In future these compounds are going to be extensively used in the development of supercomputers, telecommunication systems, light emitting diodes and semiconductor lasers ${ }^{1.22}$. GaAs has a distinct advantage in electronic speed compared with silicon and is therefore increasingly used for satellite communication systems and supercomputers. Several favourable properties as compared with silicon based devices suggest that GaAs and such other intermetallic substrates may find applications in military, space, telecommunication and supercomputing systems. All these extensive uses of GaAs will inevitably lead to an increase in the exposure of workers manufacturing these products.

\section{Possible Routes of Exposure}

Exposure to these intermetallic substrates (semiconductors) may occur during cleaning and maintenance operations for quartz glassware and during cleaning of the reactors. Exposure may also be expected during cropping, slicing, lapping, polishing, and backlapping and wafer-saving steps. Disposal of the waste products and recycling of these materials have also not yet been discussed. Exposure to airborne particulate of GaAs may therefore be potential health hazards in the 
semiconductor industry. The toxic effects of the intermetallic semiconductor materials appear to occur primarily after inhalation exposure, although oral exposure to high doses may also result in toxicity. Assessment of the risk to these workers from GaAs exposure is further complicated due to the lack of toxicity data available for this compound and, as mentioned above, is mainly regulated on the basis of inorganic arsenic toxicity. It is now well known that both GaAs and InAs dissociate into its constitutive moieties both in vitro and in vivo ${ }^{3-6)}$ and arsenic is a well-known toxic metal, so that one should not take the toxicity of these intermetallic semiconductors lightly.

Bioavailability, metabolism and excretions of most of the III-V intermetallic compounds are not well known. Bioavailability may depend on their dissolution rates and chemical properties. The reported acute lethality of these III-V intermetallic compounds is of low order. The oral $\mathrm{LD}_{50}$ of GaAs in mice and rats is more than $15 \mathrm{~g} / \mathrm{kg}$, whereas the threshold for acute effects is around $7 \mathrm{~g} /$ $\mathrm{kg}^{7}$. The threshold for acute effects (lowest published lethal dose) of inhalation of GaAs aerosol for 4 hours has been reported to be $152.5 \mathrm{mg} / \mathrm{m}^{3}$. For Indium antimonide the reported $\mathrm{LD}_{50}$ is $3.7 \mathrm{~g} / \mathrm{kg}$ by the intraperitoneal route whereas that for indium arsenide it is around $15 \mathrm{~g} / \mathrm{kg}$. For GaP the reported $\mathrm{LD}_{50}$ is $8 \mathrm{~g} / \mathrm{kg}^{7}$. The oral $\mathrm{LD}_{50}$ of metallic indium was $4200 \mathrm{mg} / \mathrm{kg}$ for rats and that of intraperitoneal and oral $\mathrm{LD}_{50}$ of $\mathrm{InP}$ was reported to be more than $5000 \mathrm{mg} / \mathrm{kg}$.

\section{Effects on Biological Systems}

The absorption of GaAs and InAs appears to be accompanied by the formation of arsenic oxide. Numerous reports have indicated that pulmonary exposure to GaAs and InAs dust represents a potential health hazard, but the degree of risk has not yet been fully defined. Recent experiments have investigated the acute toxicity of GaAs and InAs particulate fractions which are soluble under physiologically relevant in vitro conditions and produce a dose related increase in blood arsenic levels. When delivered to rats by either the oral or intratracheal route, exposure resulted in decreased body weight as well as both quantitative and qualitative changes in urinary porphyrin excretion ${ }^{7-9}$. In addition to the above, there have been a number of other studies demonstrating the solubility of GaAs particles in vivo and the tissue distribution and excretion patterns of $\mathrm{Ga}$ and As over time after the administration of GaAs particles via a variety of routes ${ }^{10 !}$. These results lead to the conclusion that particles of $\mathrm{GaAs}$ and InAs are degraded in vivo to release their constitutive elements, which are then distributed to major target organs. But Zheng et $a l .11$ indicated poor absorption of indium following repeated oral or intratracheal instillation of indium phosphide, suggesting that indium is unlikely to accumulate in the body after InP exposure. Fecal excretion is a major route for its elimination. Based on the literature available it is now well known that GaAs produces a definite adverse effect on at least three major body systems, i) pulmonary, ii) haematopoietic and iii) immune.

\section{Pulmonary Effects}

Toxicity of inhaled gallium arsenide compounds has been reported in a number of reports in the recent past ${ }^{12-15}$. It has been suggested that particulate fractions with a mean count and volume diameter of 8.3 and $12.67 \mu \mathrm{m}$ were soluble under physiologically relevant in vitro conditions $^{12}$. The intratracheal administration of this GaAs particulate fraction was relatively more toxic than equivalent doses given through the oral route and results in increased lung weight ${ }^{12)}$. Webb et al. ${ }^{12)}$ also evaluated the toxicity of $\mathrm{Ga}_{2} \mathrm{O}_{3}, \mathrm{As}_{2} \mathrm{O}_{3}$ and GaAs after the intratracheal instillation of these particles in rats. They found that the toxicity ranking was $\mathrm{GaAs}>\mathrm{As}_{2} \mathrm{O}_{3}>\mathrm{Ga}_{2} \mathrm{O}_{3}$ and suggested that the pathological responses observed in lungs were likely to be primarily due to arsenic. The lesions in lungs due to $\mathrm{Ga}_{2} \mathrm{O}_{3}$ were not remarkable. Ohyama et al. ${ }^{13)}$ observed severe lung lesions, and survival was also shortened significantly in animals intratracheally exposed to GaAs particle $(0.25 \mathrm{mg} \times 15$ times/animals) compared to controls. Goering et al. ${ }^{5 i}$ also reported histopathological changes characterised by multifocal granulomas and type II pneumocyte hyperplasia after single intratracheal instillation of GaAs $(50,100$ or $200 \mathrm{mg} / \mathrm{kg}$ ). Indium arsenide (InAs) and indium phosphide ( $\mathrm{InP}$ ) were also reported to produce histopathological changes in lungs of hamsters ${ }^{14.15}$. The major changes include alveolar and bronchiolar cell hyperplasia, pneumonia, and emphysema and metaplastic ossification after InAs exposure in rats and hamsters. Kabe et $a l .^{14)}$ using mice and oral and intraperitoneal (i.p.) routes of exposure reported a dose dependent increase in lung weight. Extramedullary granulopoiesis, eosinophillic exudates and mononuclear cells were seen in the pulmonary alveoli after i.p. administration. But the p.o. administered mice showed no clear relationship between the dose and biological effects. On the other hand, InP is reported to cause pulmonary inflammation and the particles remained in the lower airways for nearly seven days ${ }^{16.17)}$. A dose dependent increase in superoxide dismutase (SOD) and the lactate dehydrogenase (LDH) activity in the bronchioalveolar lavage fluid (BALF) was also found in InP exposed rats ${ }^{17)}$, but these studies clearly indicate adverse effects of these semiconductor materials on the pulmonary system in experimental studies. Well planned detailed studies on subjects handling these materials in the industry are still lacking. 


\section{Haematopoietic Effects}

Chemically induced disturbances of the heme biosynthetic pathway have been utilized for many years as a class of biomarkers for detecting the sublethal toxicity metals, but very few reports have indicated perturbation of heme metabolism by binary metal compounds in groups III and V. There have been a number of animal studies reported recently on the effects of GaAs on porphyrin metabolism. Goering et $a .^{5 i}$ first reported that GaAs after a single intratracheal instillation produced a dose dependent inhibition of blood delta - aminolevulinic acid dehydratase, (ALAD), an important enzyme in the heme biosynthetic pathway. They reported that the activity decreases to $5 \%$ of the control at a dose of 200 $\mathrm{mg} / \mathrm{kg}$ on day 6 after a single exposure. Urinary aminolevulinic acid (ALA) excretion was also maximum 3 to 6 days post exposure and recovered to the control value by day 18 . These authors further suggested that gallium is the primary inhibitor of ALAD after dissolution of GaAs in vivo and that competition for or displacement of zinc as the enzyme active site may be involved in the mechanism of inhibition. These data were later supported by a brief report by Flora and Das Gupta ${ }^{3}$. They reported a dose dependent inhibition of blood ALAD, whereas a moderate increase in blood zinc protoporphyrin (ZPP) and urinary ALA excretion as observed after a single oral exposure on days 1,7 and 15. A dose dependent increase in the blood arsenic concentration was also noticed, but gallium content was not detectable in animals exposed to a low oral dose of GaAs. Conner et al. ${ }^{{ }^{1}}$ recently observed that InAs and constitutive elements results in a unique urinary porphyrin excretion profile. They reported an inhibition of erythrocyte ALAD but no change in hepatic ALAD activity. In contrast renal ALAD was found to be statistically decreased by As at first but increased at a later stage after a single exposure. Studies of urinary porphyrin excretion patterns in animals treated with InAs showed marked and early $2-4$ fold increases in the excretion of penta, hexa and heptacarboxyl porphyrin at $1-5 \mathrm{~d}$, indicating that both $\mathrm{In}$ and As are biologically active after InAs exposure and that enzymes in the heme pathway such as ALAD may be very useful as markers of exposure. Nevertheless, we still strongly believe that a few more detailed studies are needed, particularly on different animal models and with various doses, but it is desirable to suggest that measurement of the activity of blood ALAD and urinary ALA excretion could be the useful early indicators of GaAs and InAs exposure. Flora et al. ${ }^{18.191}$ recently conducted a more detailed study with the rat as the experimental model and found that GaAs had a strong effect on heme synthesis but only a mild secondary effect on major physiological variables (viz., blood pressure, respiration, heart rate and neuromuscular transmission) was noticed. Furthermore, the peak adverse effects were reached at day 7 after exposure compared to observations at two other times i.e.. day 1 and 15. Previously, Webb et al. " also indicated that the urinary porphyrin concentration was changed over the $14 \mathrm{~d}$ study and accompanied by a decrease in body weight. They further concluded that the urinary uroporphyrin concentration was greater than the coproporphyrin concentration and may therefore serve as a sensitive indicator of GaAs exposure. It is therefore evident from the abovementioned studies that these semiconductor intermetallic substrates affect the heme synthesis pathway and they may also serve as an early indicator of toxicity. But in order to be able to suggest a more specific and sensitive indicator, further detailed studies are definitely required in this area.

\section{Immunological Effects}

A number of recent reports on laboratory animals have demonstrated that the immune system is also one of the sensitive target sites after GaAs exposure. Both humoral and cell mediated sites of immunity are specifically affected $^{20}$ and all cell types involved in the generation of a $\mathrm{T}$ cell dependent antibody response are functionally compromised $d^{21.22)}$

The immunotoxic action of GaAs was found to occur within the first $36 \mathrm{~h}$ of an immune response and was shown to be a result of the toxic action of the arsenic component of $\mathrm{GaAs}^{19,23)}$. In addition, GaAs targets several $\mathrm{T}$ cell mediated immunological functions including, but not limited to, the DHR, the CTL response, the MLR and the $T$ cell dependent humoral immune response ${ }^{20.221}$. A few other recent studies have revealed that after $\mathrm{GaAs}$ exposure $T$ cell proliferation was selectively targeted and that this was likely due to effects on the IL-2 receptor; the receptor for the major $\mathrm{T}$ Cell growth factor ${ }^{241}$.

The exact biochemical or molecular mechanism by which GaAs produces immunosuppression is not known, although several possibilities have been proposed ${ }^{21 .}{ }^{24}$. It is now generally believed that arsenic dissociation from GaAs may be responsible for some of the immunotoxic effects and may constitute a potential risk to workers exposed to this compound ${ }^{24}$.

The above studies therefore confirm that the immune system is a sensitive target organ for toxicity from GaAs and that such suppressive effects may play a role in the observed rate of semiconductor worker absenteeism due to illness ${ }^{251}$. Data regarding the effect of InAs on the immune system are lacking.

\section{Reproductive Effects}

A few recent studies by Omura and his group found that the repetitive intratracheal instillation of GaAs decreased the sperm count and increased the proportion of abnormal sperm in the epididymis of rats ${ }^{26)}$. though the effects of $\mathrm{GaAs}$ on spermatogenesis in the testis were 
not clearly demonstrated. This group recently published a more comprehensive report in which the testicular toxicity of GaAs and InAs was examined in rats by repeated intratracheal instillation of these substances in suspension twice a week, 16 times altogether ${ }^{27}$. They reported a significant decrease in sperm counts and significant increase in the proportion of morphologically abnormal sperm in the epididymis of the GaAs exposed group. It was indicated that $\mathrm{GaAs}$ disturbed the spermatid head transformation at the late spermiogenic phases and caused spermiation failure ${ }^{27}$. InAs caused a sperm count decrease in the epididymis, though its testicular toxicity was relatively weak compared to with that of GaAs. Arsenic trioxide, a probable dissolution product of $\mathrm{GaAs}$ and InAs in vivo did not show any sign of testicular toxicity in this study. It could therefore be suggested that arsenic, gallium and indium play a role in the testicular toxicity of GaAs and InAs ${ }^{27)}$. In another recently reported interesting study, these authors confirmed that among these metals, gallium might play a main role in the testicular toxicity of GaAs in hamsters ${ }^{28}$. They reported that the serum arsenic concentration in GaAs treated hamsters was less than half of that in arsenic trioxide treated hamsters in which no lesticular toxicity was observed. It is recommended that further studies be conducted in order to examine the in vivo distribution of arsenic, gallium and indium in the testis to evaluate the degree of contribution of these elements to the testicular toxicity of GaAs and InAs.

\section{Renal Effects}

Goering et al. for the first time reported that intratracheal exposure to GaAs particles could lead to both ultrastructural and biochemical manifestation of renal tubular injury. Flora et al ${ }^{(9)}$ reported an increase in urinary protein excretion and renal alkaine phospharase activity but the change were dose dependent. A few subsequent studies also showed marked alterations in renal tubule gene expression after in vivo administration of $\mathrm{GaAs}^{29}$. Conner et al. ${ }^{29}$ and Conner ${ }^{20)}$ showed marked alterations in renal tubule gene expression after GaAs and InAs exposure. Indium administration was also reported to decrease overall protein synthesis, which is consistent with the renal accumulation of $\mathrm{In}^{7}$.

\section{Hepatic Effects}

Webb et al. ${ }^{101}$ reported impaired liver function due to arsenic dissociated from GaAs as indicated by increased urinary excretion of uroporphyrin. Flora ${ }^{31 !}$ also recently reported changes in some key biochemical variables in the liver of rats exposed to various doses of GaAs, but the changes were only mild. The liver has been reported to be a target for indium ${ }^{321}$ and arsenate ${ }^{331}$, but there has been no information so far in the literature about the effect of InAs on the liver.

\section{Central Nervous System Effects}

There have been no detailed studies conducted so far on the effects of $\mathrm{GaAs}$ on the central nervous system (CNS), but Flora $e t a l^{34)}$ demonstrated changes in the steady state levels of some brain neurotransmitters. The results indicated only a moderate effect of GaAs, after repeated low level exposure, on the level of brain biogenic amines (dopamine, norepinephrine and 5-hydroxytryptamine) but a significant effect on brain and blood acetylcholinesterase (AChE) activity. Histopathological observations also revealed some mild effects, particularly in the cerebral cortex region.

\section{Other Effects}

There are no reports of effects of these semiconductor materials on other organ systems, but it is now clear beyond any doubt that GaAs and InAs particles dissolve and release arsenic. It is likely that chronic low level exposure may ultimately lead these agents to produce clinical manifestation in worker populations.

Flora et al. ${ }^{18)}$ reported few changes, particularly at a higher dose level, in the physiological variables, viz. blood pressure, heart rate, respiration and twitch response. The peak adverse effects were noticed at day 7 after a single exposure compared to observation at two other times (i.e., day 1 and day 15).

\section{Biological Monitoring}

There is so far no well-conducted report available in the literature about the biological monitoring of subjects handling these compounds. Yamamuchi et al. ${ }^{357}$ reported a method for biological monitoring of inorganic arsenic exposure, and the chemical species of arsenic were measured in the urine and the hair of GaAs plant workers and copper smelter workers. It was revealed that the total arsenic concentration in the hair of all groups of GaAs plant workers tended to be higher than in the control groups. It is suggested that urinary arsenic levels be generally used as a biologic monitor for arsenic exposure. It is recommended that due to a possible high arsenic concentration in sea-food, the workers should be asked to refrain from eating sea food 3 4 days before being tested for arsenic in a GaAs or InAs plant. Tests for an inorganic form of arsenic in blood, urine and hair along with determination of blood ALAD and urinary ALA excretion may be conducted as a routine measure to determine arsenic exposure in such industries.

No definite data are available and no specific exposure limits have been formulated for any of the III-V intermetallic compounds. The National Institute of Occupational Safety and Health (NIOSH) issued an alert and recommended that exposure to GaAs be controlled by observing the NIOSH Recommended Exposure Limit for inorganic arsenic ( $2 \mu \mathrm{g} / \mathrm{m}^{3}$ of air as a 15 min ceiling). NIOSH also recommended that the concentration of $\mathrm{GaAs}$ in air be estimated by the determination of arsenic ${ }^{12 !}$. 


\section{Treatment}

As the toxicology of GaAs is still not very well understood or clearly defined, the treatment also remains doubtful. British Anti Lewisite (2,3-dimercaprol; BAL) has been used for the treatment of poisoning by arsenic compounds ${ }^{36}$, but this compound has many disadvantages such as a low safety ratio, unpleasant side effects and difficulty in systemic administration. Two recent reports have indicated in animal models that treatment with meso 2,3-dimercaptosuccinic acid (DMSA) and sodium 2,3dimercaptopropane 1-sulfonate (DMPS) may reverse most of the immunosuppressive effects ${ }^{37.38}$. Attempts are also being made in our laboratory to synthesise and evaluate mono and diesters of DMSA for treating chronic low level GaAs exposure. Preliminary results indicate a beneficial role of monoisoamyl DMSA in treating GaAs exposure (Flora et al. unpublished results). Another possible approach to chelation treatment is combination therapy with an essential metal or an antioxidant as an adjuvant during chelating agent administration. We recently reported that selenium administration during GaAs exposure has some preventive value particularly against the altered immunological and haematopoietic effects ${ }^{3 y}$. In a first study of its kind we also reported that combined administration of $\mathrm{N}$-acetyl cystein during treatment with meso 2,3-dimercaptosuccinic acid (DMSA) leads to a more pronounced elimination of arsenic from the soft tissues and recovery in the altered biochemical variables indicative of oxidative stress $s^{401}$, but these data are only from experimental models and not supported by evidence in any human case study.

\section{Future Research Trends}

Further research is urgently required in the following important areas:

- There is a need to develop biological indicators that are useful, more specific and reliable under conditions of multielement exposure.

- There is a need to understand the mechanism and metabolism of action of cell injury so that the indicator response can be correctly interpreted.

- It is also of great importance to investigate the role of various factors such as age, sex, physiological states, such as pregnancy, and nutritional status, which may influence the toxic manifestation of these intermetallic compounds.

- Lastly, there is an urgent need to develop safe, highly effective and specific antidotes for treating cases of possible acute or chronic GaAs/InAs exposure.

\section{Conclusion}

As is evident from the few above mentioned studies, these intermetallic semiconductor materials possess toxic biological properties and this may lead to potential occupational and environmental health consequences. Therefore, in order to prevent such hazards associated with the handling of these compounds, detailed studies need to be conducted in several areas viz. target organ toxicity, mechanisms of action, specific biological indicators, preventive and therapeutic measures, besides possible health monitoring of subjects handling these compounds.

Acknowledgment: The author thanks Dr. R.V. Swamy, Director of the establishment, for his support and encouragement.

\section{References}

1) Robinson AL. GaAs readied for high-speed microcircuit. Science 1983; 219: 275-277.

2) Fowler BA. Toxic metals in emerging technologies. In Metal Toxicology, edited by RA Goyer, CD Klaassen. MP Waalkes, Academic Press, NY, 1985: 187-196.

3) Flora SJS, Gupta SD. Effects of single exposure to gallium arsenide on some biochemical variables in porphyrin metabolism in rats. J Appl Toxicol 1992; 12: 333-334.

4) Flora SJS, Gupta SD. Toxicology of gallium arsenide: an appraisal. Def Sci J 1994; 44: 5-10.

5) Goering PL, Maronpot RR, Fowler BA. Effect of intratracheal gallium arsenide administration on aminolevulinic acid dehydratase in rats: relationship to urinary excretion of aminolevulinic acid. Toxicol Appl Pharmacol 1988; 92: 179-193.

6) Yamamuchi H, Takahashi K, Yamamura Y. Fowler BA. Metabolism of subcutaneous administered indium arsenide in the hamster. Toxicol Appl Pharmacol 1992; 116: 66-70.

7) Carter DE, Sullivan JB. Intermetallic semiconductor and inorganic hydrides. In: Hazardous Materials Toxicology, Clinical Principles of Environment, Sullivan JB, Kreiger GR, eds. Williams \& Wilkins, MD, USA, 1992: 916-921.

8) Conner EA, Yamamuchi H, Fowler BA. Alterations in the heme biosynthetic pathway from the III-V semiconductor metal, indium arsenide (InAs). $\mathrm{Chcm}$ Biol Interact 1995; 96: 273-285.

9) Webb DR, Sipes IG. Carter DE. In vitro solubility and in vivo toxicity of gallium arsenide. Toxicol Appl Pharmacol 1984: 76: 96-104.

10) Yamamuchi H, Takahashi K, Yamamura Y. Metabolism and excretion of orally and intraperitoneally administred gallium arsenide in the hamsters. Toxicology 1986; 40: 237-246.

11) Zheng W, Winter SM, Kattnig MJ, Carter DE, Sipes IG. Tissue distribution and elimination of indium in male Fischer 344 rats following oral and intratracheal administration of indium phosphide. J Toxicol Environ Health 1994; 43: 483-494.

12) Webb DR, Wilson SE, Carter DE. Comparative pulmonary toxicity of gallium arsenide, gallium (III) oxide or arsenic (III) oxide intratracheally instilled into rats. Toxicol Appl Pharmacol 1986; 82: 405-416. 
13) Ohyama S, Ishinishi N, Hisangawa A, Yamamoto A. Comparative chronic toxicity including tumorigenicity of gallium arsenide and arsenic trioxide intratracheally instilled into hamsters. Appl Organomet Chem 1988; 2: 233-237.

14) Kabe I, Omae K, Nakashima H, et al. In vitro solubility and in vivo toxicity of indium phosphide. J Occup Health 1996; 38: 6-12.

15) Tanaka A, Hisanaga A, Hirata M, Omura M, Makita $Y$, Inoue N, Ishinishi N. Chronic toxicity of indium arsenide and indium phosphide to the lungs of hamsters. Fukuoka Igaku Zasshi 1996; 87: 105-115.

16) Uemura T, Oda K. Omae K, et al. Effects of intratracheally administered indium phosphide on male Fischer 344 rats. J Occup Health 1997; 39: 205-210.

17) Oda K. Toxicity of a low level of indium phosphide $(\mathrm{InP})$ in rats after intratracheal instillation. Indust Health 1997; 35: 61-68.

18) Flora SJS, Dube SN, Vijayaraghvan R, Pant SC. Changes in certain hematological and physiological variables following single gallium arsenide exposure in rats. Biol. Trace Elem Res 1997; 58: 197-208.

19) Flora SJS, Kumar P, Kannan GM, Rai GP. Acute oral gallium arsenide exposure and changes in certain hematological, hepatic, renal and immunological indices at different time intervals in male wistar rats. Toxicol Letters 1999; 94: 103-113.

20) Sikorski EE, McCay JA, White KL. Bradley SG, Munson AE. Immunotoxicity of the semiconductor gallium arsenide in female B6C3F 1 mice. Fund Appl Toxicol 1989; 13: 843-858.

21) Sikorski EE, Burns LA, McCay KL, Stern M, Munson AE. Suppression of splenic accessory cell function in mice exposed to gallium arsenide. Toxicol Appl Pharmacol 1991; 110: 143-156.

22) Sikorski EE, Burns LA, Stern ML, Luster MI, Munson AE. Splenic cell target in gallium arsenide induced suppression of the primary antibody response. Toxicol Appl Pharmcol 1991; 110: 129-142.

23) Burns LA, Sikorski EE, Saady JJ, Munson AE. Evidence for arsenic as the immunosuppressive component of gallium arsenide. Toxicol Appl Pharmacol 1991; 110: 157-169.

24) Burns LA, Munson AE. Reversal of gallium arsenide induced suppression of the in vitro generated antibody response by supernatants from vehicle exposed cultures I. pharmacokinetic following in vitro and in vivo exposure. J Pharmacol Exp Ther 1993; 265: 144-149.

25) Ladou J. Health issues in the global semiconductor industry. Ramazini Ann 1992; 3: 110-112.

26) Omura M, Tanaka A. Zhao M, Hirata M, Makita Y, Inoue $\mathrm{N}$, Gotoh $\mathrm{K}$. Toxic effects of gallium arsenide on sperm in rats by repeated intratracheal instillations. J Occup Health 1995; 37: 165-166.

27) Omura M, Tanaka A, Zhao M, Hirata M, Makita $Y$, Inoue N, Ishinishi N. Testicular toxicity of gallium arsenide, indium arsenide and arsenic oxide in rats by repetitive intratracheal institlation Fund Appl Toxicol 1996; 32: 72-78.
28) Omura M, Hirata $M$, Tanaka $A$, et al. Testicular toxicity evaluation of arsenic containing binary compound semiconductor semiconductor, gallium arsenide and indium arsenide in hamsters. Toxicol Lett 1996; 89 : $123-129$.

29) Conner EA, Yamamuchi H, Fowler BA, Akkarman M. Biological indicators for monitoring exposure/toxicity from III-V semiconductors J Exp Anal Environ Epidemiol 1993; 314: 431-440.

30) Conner EA. Control methods for metals and metalloids in III-V materials vapor phase epitaxy. In: Proceedings of the Conference on Hazards Assessment and Control Technology in Semiconductor Manufacturing. American Council of Government Industrial Hygieniest; Lewis, Chelsea MI: 1989; 243-258.

31) Flora SJS. Alterations in some hepatic biochemical variables following repeated gallium arsenide administration in rats. Int Hepatol Comm 1996; 5: $97-$ 103.

32) Fowler BA. Indium. In: Friberg L, Nordberg GF, Vouk VB, eds. Handbook on the toxicology of metals. 2nd edition, Amsterdam; Elsevier, 1986: 939-944.

33) Fowler BA, Wood JS. The effects of prolonged oral arsenate exposure on liver mitochondria of mice: Morphometric and biochemical studies. Toxicol Appl Pharmacol 1979; 50: 177-187.

34) Flora SJS, Dube SN, Jant SC, Sachan AS. Effect of multiple gallium arsenide exposure on some biochemical alterations in rat brain. Ind Health 1994; 32: 247-252.

35) Yamamuchi H, Takahashi K, Mashiko M, Yamamura Y. Biological monitoring of arsenic exposure of gallium arsenide and inorganic arsenic exposed workers by determination of inorganic arsenic and its metabolites in urine and hair. Am Ind Hyg Assoc J 1989; 50: 606612.

36) Hammond PB, Bieliles RP, Klaassen CD, Amdur MO, Doull J. In: Klaassen CD, Amdur MO, Doull J, eds. Toxicology: The Basic Science of Poisons. NY: MacMillan Publishing Co, 1986.

37) Burns LA, Butterworth LF, Munson AE. Reversal of gallium arsenide-induced suppression of the antibody response by a mixed disulfide metabolite of meso 2,3 dimercaptosuccinic acid. J Pharmacol Exp Ther 1993; 264: 695-700.

38) Flora SJS, Kumar P. Biochemical and immunotoxicological alterations following repeated gallium arsenide exposure and their recoveries by meso 2,3-dimercaptosuccinic acid and 2,3dimercaptopropane 1-sulfonate administration in rats. Environ Toxicol Pharmacol 1996; 2: 315-320.

39) Flora SJS, Kannan GM, Kumar P. Selenium effects on gallium arsenide induced biochemical and immunotoxicological changes in rats. Chem Biol Interact 1999; 122: 1-13.

40) Flora SJS. Arsenic induced oxidative stress and its reversibility following combined administration of $\mathrm{N}$ acetyl cysteine and meso 2,3-dimercaptosuccinic acid in rats. Clin Exp Pharmacol Physiol 1999; 26: 865-869. 